\title{
Compatibility Relations For Identity Analytics
}

\author{
Harry Katzan, Jr., Savannah State University, USA
}

\begin{abstract}
Methods for categorizing certain subjects, based on belief structures, are an important aspect of modern society. In a related paper, we present a method for combining belief, attributable to diverse knowledge sources, in order to obtain a measure of group membership. The scope of group identity is much greater than digital security and affects other societal endeavors. In this paper, we are going to propose methods for propagating belief through a complex network of belief assessments. The methods are known as compatibility relations. The related paper is "Identity Analytics and Belief Structures," published in an earlier issue of the Journal of Business \& Economics Research.
\end{abstract}

Keywords: Identity; belief; compatibility relation; belief propagation

\section{INTRODUCTION}

dentity refers to the categorization of an individual and the assigning of a name to that determination. We are going to approach the subject of identity from a data analytic viewpoint, where the primary challenge is to use identity in an effective way to determine group membership. Methods have been developed for propagating belief through a complex network of belief assessments. The focus of this paper is on compatibility relations, defined as the mapping of belief between identity namespaces. This paper is intended to accompany a related paper entitled "Identity Analytics and Belief Structures." (Katzan 2010) The introductory section contains common material, so it can be read independently.

\section{Identity Concepts}

Identity is a means of denoting an entity in a particular namespace and is the basis of analytic behavior analysis. We are often afforded the identity of a person based on the judgment of a third party and are obligated to respond to that assessment. It would seem to be prudent in a civilized society to obtain additional information on the subject and combine the various items of information to obtain a composite view before engendering a timely response to the situation.

Identity is primarily used to establish a relationship between an attribute or set of attributes and a person, object, event, concept, or theory. The relationship can be direct, based on physical evidence, and in other cases, the relationship is indirect and based on a reference to other entities. Direct knowledge is known by acquaintance and is evidenced by a physical connection. Indirect knowledge is determined through a reference to a particular with which the analyst is acquainted. This form is known as knowledge by description. (Russell 1912) Direct knowledge is determined through sense data, memory, or introspection. Indirect knowledge is determined through a reference to another particular, as in "the person who was mayor in 2009."

\section{Belief Concepts}

Belief is often regarded as a mental state in which a person holds a proposition to be true without necessarily being able to prove its truth to other persons. Even though absolute certainty is not required with belief, a person's set of beliefs can play an important role in the causation of behavior. If a representation for belief $\mathrm{P}$ exists in a person's mind, then it is an explicit belief. If a representation for belief Q does not exist in a person's 
mind but is based on another proposition $\mathrm{P}$, then it is an implicit belief. Beliefs that are based on an associative relationship are usually regarded as implicit beliefs.

Dispositional belief refers to the supposition that the subject is disposed to possess a certain stance on a topic or is inclined to a particular behavior. Occurent belief refers to the assumption that the subject is actually performing a sequence of actions. The penultimate example is also an example of dispositional belief. Direct knowledge, or information obtained from a trusted source, that a subject is performing a certain action is associated with occurent belief. In the latter case, verification of identity may be of some concern and be the difference between "belief in" and "knowledge of."

\section{UNCERTAINTY}

Identity and uncertainty are related within the domain of identity analytics and belief propagation. When an analyst believes something but cannot prove it analytically or statistically, he or she is assigning a measure to the strength of the evidence supporting a specific proposition. In the present context, we are interested in the certainty of group membership.

\section{Analytic Behavior}

Analytic behavior is based on two related but often conflicting theories of action. At the individual level, people think in a cause-oriented fashion and use information to match existing patterns in order to make sense out of a minimal amount of information. (Campbell 1989, Tversky 1974) The mind too easily forms prototypes and constructs scenarios. At the organizational level, operational knowledge, based on a partial mapping of reality, is developed to determine how an organization responds to external stimuli and generates strategies and actions. Thus, both individuals and organizations do not typically handle uncertainty but respond to it according to existing ideologies. Implicit in this discussion is the not-so-obvious fact that we often have too much information and have a tendency to relate it to existing scenarios regardless of their applicability. In order to execute assessment in a judicious manner, a cognitive process, comprised of selection, processing, and response, is proposed as a context for identity analysis based on uncertainty.

Two forms of information are required to handle uncertainty: indicators from the real world and belief in what those indicators mean. Collectively, the combination of indicators, belief, and meaning is known as evidence. Evidence from independent knowledge sources should be combined to construct effective courses of action, as denoted by the lifecycle for belief revision and organizational action. (Katzan 1992)

Based on the degree to which a phenomenological system is accessible to empirical investigation, three modalities have been established: (Sutherland 1975)

- $\quad$ Empirico-inductive modality in which facts can be determined from observation and principles can be derived from facts.

- $\quad$ Hypothetico-deductive modality in which hypotheses are based on intuition, imagination, and other intellectual methods and deductive methods are used to verify predictions with observations.

- $\quad$ Knowledge-based modality that is characterized by key indicators serving as access keys to information structures and response scenarios, as in medical diagnosis, wherein knowledge structures are organized as a complex network of relationships.

Although identity analysis would essentially employ the three modalities, as required, the emphasis would necessarily focus on the knowledge-based modality, since indicators can certainly give some insight into the true state of reality - but not always with certainty.

\section{Uncertain Reasoning}

Most information systems treat information as though it was a proven fact, but in actuality, this is rarely the case. In addition, inferences based on so-called exact information are equally uncertain. Valid inference relations 
should take the form:

\section{if $x$ then $y$ with confidence $m$}

where the confidence factor $m$ reflects the degree of confidence that a domain expert assigned to the inference relation. The uncertainty in this case is the reflection of one or more of the following conditions: (1) Inherent uncertainty in the possibility set; (2) Incomplete evidence; and (3) Incorrect evidence because of measurement errors. The uncertainty, incompleteness, and incorrectness of information are the reasons we wish to model identity information from the viewpoint of uncertain reasoning.

\section{Degrees of Belief}

In order to investigate degrees of belief, we are going to employ the following construct:

if $x$ then $y$ (to degree $a$ )

and refer to the expression as an inference rule. Here are some examples:

if the patient has a large lump

then there is evidence (0.7) that medical tests are necessary.

if housing starts are up

then there is evidence (0.6) that interest rates will also rise within 6 months.

The certainty factor in an inference rule is an expression of its inherent uncertainty. There is frequently some uncertainty in the antecedents, as well, because of reasons given in the previous section. This prospect is demonstrated in the following example:

if the patient has a large lump (0.7)

and the patient's blood is weak $(0.4)$

then there is strong evidence (0.9) that the patient should go to the hospital for treatment.

In this example, the evidence of a large lump is uncertain (0.7) because of the vagueness of the word "large," and similarly with the evidence for "weak" blood (0.4). The certainty associated with the rule is, in this case, equal to (0.9).

In the antecedent part of a rule, evidence is combined in accordance with the following definitions for the logical operators:

\begin{tabular}{|c|c|c|}
\hline $\begin{array}{l}p \text { and } q \\
p \text { or } q \\
\text { not } p\end{array}$ & $\begin{array}{l}=\min (p, q) \\
=\max (p, q) \\
=1-p\end{array}$ & $\begin{array}{l}\text { The smaller } \\
\text { The larger } \\
\text { The inverse }\end{array}$ \\
\hline
\end{tabular}

The rules are then evaluated according to the following steps:

1. If the antecedent is a logical expression, then it is evaluated as covered.

2. The belief for the conclusion produced by a rule is the belief for the antecedent multiplied by the certainty associated with the rule.

3. The belief for a fact produced as the conclusion of one or more rule evaluations is the maximum of the beliefs produced by all of the rules that yield that conclusion. 
Example:

Rule 1

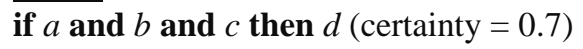

Rule 2

if $h$ or $i$ then $d$ (certainty $=0.8$ )

Assume that facts $a, b, c, h$, and $i$ have beliefs of $0.7,0.3,0.5,0.7$, and 0.9 , respectively. The following computation produced a belief of 0.72 for $d$ :

$\min (a, b, c)=\min (0.7,0.3,0.5)=0.3 \quad$ By step 1

Belief for rule $1=0.3 \times 0.7=0.21 \quad$ By step 2

$\max (h, i)=\max (0.7,0.9)=0.9$ By step 1

Belief for rule $2=0.9 \times 0.8=0.72 \quad$ By step 2

$\max ($ Belief for rule 1, Belief for rule 2) $=0.72 \quad$ By step 3

This is the inference method used for possibility theory (Fuzzy Set Theory). (Zadeh 1986) The results are not confirmatory but illustrate the gist of belief propagation.

\section{Assignment of Belief}

The assignment of belief involves the association of a basic probability assignment to the uncertainty inherent in a mutually exclusive and exhaustive set of possibilities. In some instances, the assignment of belief can be made by using the probability values in another probability space. (Neapolitan 1990)

Consider the task of determining the price of equity DS. Let R stand for the proposition, "The value of DS will rise," and let S represent the proposition, "Sam predicts the value of DS will rise." It would be desirable to have the conditional probability of $\mathrm{R}$ given $\mathrm{S}$, i.e., $\mathrm{P}(\mathrm{R} \mid \mathrm{S})$, but that information is unfortunately not available through repeated trials.

On the other hand, Sam is a crackerjack analyst, and given that he has performed a thorough fundamental analysis of an equity, it will unquestionably rise if Sam says it will. However, Sam is a busy guy, and coupled with the fact that Sam has quite an ego, the situation sometimes results in Sam giving an investment opinion off the cuff, when he hasn't done his homework. Assume that Sam does his homework 70\% of the time and let H represent the proposition, "Sam has done his homework." Thus, $\mathrm{P}(\mathrm{H})=0.70$ in this instance.

We are seeking the probability of $\mathrm{R}$ and can use the probabilities in another probability space to infer it. Now if $\mathrm{H}$ is true, then events $\mathrm{H}$ and $\mathrm{R}$ are compatible and we have the relationship:

$\mathrm{m}_{1}(\{\mathrm{R}\})=\mathrm{P}(\mathrm{H})=0.7$

where $\mathrm{m}_{1}$ represents the mass afforded to the enclosed proposition. (Katzan 1992, 2008, 2010) The other 30\% of the time, Sam takes an educated guess, so all bets are off. Thus, the complement of $\mathrm{H}$, namely $\mathrm{H}^{\mathrm{c}}$, is compatible with $\mathrm{R}$ and $\mathrm{R}^{\mathrm{c}}$, so we have:

$\mathrm{m}_{1}\left(\left\{\mathrm{R}, \mathrm{R}^{\mathrm{c}}\right\}\right)=\mathrm{P}\left(\mathrm{H}^{\mathrm{c}}\right)=0.3$

In this instance, Sam has predicted that DS will rise, i.e., proposition S, and Sam has done his homework. Thus, H and $\mathrm{R}^{\mathrm{c}}$ are not compatible. We have used Sam's probability space of $\mathrm{H}$ to determine DS's probability space for $\mathrm{R}$.

We can use a similar argument to obtain a second estimate over the frame of discernment $\left\{R, R^{c}\right\}$. Suppose that $\mathrm{R}$ is true when the value of the Dow rises, representing the latter proposition by D. Assume the Dow goes up $80 \%$ of the time; when it does not, it's another random walk down Wall Street. Thus, we have the 
relationships:

$\mathrm{m}_{2}(\{\mathrm{R}\})=\mathrm{P}(\mathrm{D})=0.8$

$\mathrm{m}_{2}\left(\left\{\mathrm{R}, \mathrm{R}^{\mathrm{c}}\right\}\right)=\mathrm{P}\left(\mathrm{D}^{\mathrm{c}}\right)=0.2$

As before, $D$ is compatible with $R$ but not with $R^{c} ; D^{c}$ is compatible with both $R$ and $R^{c}$. Using Dempster's rule of combination, we obtain a composite picture of the situation and demonstrate the compatibility relation:

$\mathrm{m}=\mathrm{m}_{1} \oplus \mathrm{m}_{2}$

$\mathrm{m}(\{\mathrm{R}\})=0.94$

$\mathrm{m}\left(\left\{\mathrm{R}, \mathrm{R}^{\mathrm{c}}\right\}\right)=0.06$

Information on Dempster-Shafer Theory is given in the accompanying paper (Katzan 2010) and in the relevant publications. (Shafer 1976, Dempster 1967, and Katzan 1992)

\section{COMPATIBILITY RELATIONS}

This section gives an algorithm for exercising a compatibility relation between focal sets in two information spaces. A belief system can be conveniently conceptualized as a directed graph $(\mathrm{G})$, represented symbolically as $\mathrm{G}=\{\mathrm{V}, \mathrm{E}\}$, where $\mathrm{V}$ is a set of vertices and $\mathrm{E}$ is a set of edges. The vertices are called nodes and the edges are referred to as links. The nodes store information that take the form of belief structures. The links represent the relationship between nodes. A belief system is a collection of four entities: evidence, nodes, links, and decision scripts.

\section{Evidence}

An element of evidence is represented as a simple support function over a frame of discernment and regarded as a "cloud of evidence" that takes the form:

$\left.\mathrm{e}=\left\{\mathrm{obj}_{1}, \mathrm{obj}_{2}, \ldots, \mathrm{obj}_{n}\right), \mathrm{bpa}\right\}$

where obj $j_{i}$ is an object from the frame of discernment and bpa is a basic probability assignment taken as a measure of belief that a random variable is contained in the belief set. If $\Theta$ is the frame of discernment, then $m(\mathrm{~A})$ is a measure of belief assigned to a subset $\mathrm{A}$ of $\Theta$.

\section{Nodes}

A node is an abstract structure that serves to hold evidence. In general, there are two types of nodes: those that are linked to the environment and those that are not. Three classes exist:

- Affector nodes that obtain their information from clouds of evidence. If the input to an affector node is a single cloud, then that affector node holds a simple support unit. If there are two or more inputs to an affector node, then that evidence is combined using Dempster's rule of combination, and the node holds a separable support unit. An affector node represents an explicit belief.

- $\quad$ Constructor nodes that exist as internal nodes and have belief propagated to them and also have belief propagated from them. If belief is propagated to a constructor node from two or more nodes, then it is always combined using Dempster's rule. A constructor node represents an implicit belief.

- Effector nodes that are objects to which external entities are attached, such as with a decision script. An effector node is a means of taking appropriate action if specified conditions are met.

A node may be assigned an initial state and takes the meaning of an explicit belief. 


\section{Links}

A link represents a direction along which belief can be propagated. Consider two frames of discernment $\Theta^{\mathrm{A}}$ and $\Theta^{\mathrm{B}}$. Further, assume two corresponding sets of propositions $\left\{\mathrm{P}_{j}^{\mathrm{A}}\right\}$ and $\left\{\mathrm{P}_{j}^{\mathrm{B}}\right\}$, where $j=1,2, \ldots, m$ and $k=1,2, \ldots, n$. The relation

$\Theta_{j}^{\mathrm{A}} \rightarrow \Theta_{k}^{\mathrm{B}}$

denotes a linking of frames $\Theta^{\mathrm{A}}$ and $\Theta^{\mathrm{B}}$ through a set of rules of the form, if $\mathrm{p}_{j}$ then $\mathrm{p}_{k}$ for couplets $\mathrm{p}_{j}^{\mathrm{A}}$ and $\mathrm{p}_{k}{ }^{\mathrm{B}}$ from frames $\Theta^{\mathrm{A}}$ and $\Theta^{\mathrm{B}}$, respectively.

The set of couplets and a probability assignment of the form

$r_{\mathrm{B}}{ }^{\mathrm{A}}=\left(\left(\left(\mathrm{p}_{1}{ }^{\mathrm{A}}, \mathrm{p}_{1}{ }^{\mathrm{B}}\right)\left(\mathrm{p}_{2}{ }^{\mathrm{A}}, \mathrm{p}_{2}{ }^{\mathrm{B}}\right)\left(\mathrm{p}_{3}{ }^{\mathrm{A}}, \mathrm{p}_{3}{ }^{\mathrm{B}}\right) \ldots\right) \operatorname{prob}_{\mathrm{B}}{ }^{\mathrm{A}}\right)$

is known as a "compatibility relation." $\Theta^{\mathrm{A}}$ and $\Theta^{\mathrm{B}}$ are not necessarily distinct.

\section{Decision Scripts}

A decision script translates a resultant belief structure into a prescription for action. We are going to define an inference relation of the form

(antecedent, threshold) $\rightarrow$ (consequent, certainty-value)

It should be interpreted as follows: if the bpa of the antecedent exceeds the threshold, replace the antecedent with the consequent and compute its $b p a$ (i..e., the new $b p a$ ) as the product of the antecedent's bpa and the consequent's certainty-value. For example, consider the inference relation

$(\{a, b\}, 0.6) \rightarrow(\{c, d\}, 0.7)$

Thus, antecedent of $\{\{a, b\}, 0.8\}$ would map to a consequent of $\{\{c, d\}, 0.56\}$.

\section{Technical Description}

In a compatibility relation, a set of rules of the form

if $\mathrm{p}_{j}^{\mathrm{A}}$ then $\mathrm{p}_{k}^{\mathrm{B}}$

along with a basic probability assignment is represented as

$r_{\mathrm{B}}{ }^{\mathrm{A}}=\left(\left(\left(\mathrm{p}_{1}{ }^{\mathrm{A}}, \mathrm{p}_{1}{ }^{\mathrm{B}}\right)\left(\mathrm{p}_{2}{ }^{\mathrm{A}}, \mathrm{p}_{2}{ }^{\mathrm{B}}\right)\left(\mathrm{p}_{3}{ }^{\mathrm{A}}, \mathrm{p}_{3}{ }^{\mathrm{B}}\right) \ldots\right) \operatorname{prob}_{\mathrm{B}}{ }^{\mathrm{A}}\right)$

Each couplet of the form $\left(\mathrm{p}_{j}^{\mathrm{A}}, \mathrm{p}_{k}^{\mathrm{B}}\right)$ denotes that an element $\mathrm{p}_{j}{ }^{\mathrm{A}}$ in $\Theta^{\mathrm{A}}$ is replaced by the element $\mathrm{p}_{k}{ }^{\mathrm{B}}$ in $\Theta^{\mathrm{B}}$, denoting the aforementioned mapping from $\Theta^{\mathrm{A}}$ to $\Theta^{\mathrm{B}}$.

For example, consider two frames of discernment $F_{1}$ and $F_{2}$ delineated as follows:

$\mathrm{F}_{1}=\{\mathrm{a}, \mathrm{b}, \mathrm{c}\}$

$\mathrm{F}_{2}=\{\mathrm{x}, \mathrm{y}, \mathrm{z}, \mathrm{w}\}$

A focal set defined on $F_{1}$ is

$\mathrm{F}_{1}=((\mathrm{a}, \mathrm{b}), 0.6)$ 
Consider the set of rules

\section{if a then $x$ \\ if a then $y$ \\ if $b$ then $w$ \\ if $\mathrm{c}$ then $\mathrm{y}$ \\ if $c$ then $z$}

with a measure of belief of 0.8 . This is equivalent to stating the belief that one or more of the rules in the set would hold with a subjective probability of 0.8 . The set of rules is expressed as a compatibility relation of the form:

$r=((\mathrm{a}, \mathrm{x}),(\mathrm{a}, \mathrm{y}),(\mathrm{b}, \mathrm{w}),(\mathrm{c}, \mathrm{y}),(\mathrm{c}, \mathrm{z})), 0.8)$

The simple compatibility relation $r$ is applied to the focal set $\mathrm{F}_{1}$ by replacing each element of $\mathrm{F}_{1}$, that is elements $a$ and $b$, by the set of consequents of all rules in which that element is equal to its antecedent. The process is summarized as follows:

1. For each focal element of $\mathrm{F}_{1}$, i.e., $a$ or $b$, replace it with the second element of all couplets where the first element matches. Thus, $a$ is replaced with $x$ and $y$, and $b$ is replaced with $w$, yielding the focal set $(x, y, w)$ over $\mathrm{F}_{2}$.

2. Take the product of the probabilities, i.e., $0.6 \times 0.8$, as with Dempster's rule.

The sequence of operations yields the support unit

$((x, y, w), 0.48)$

which is the projection of $F_{1}$ onto $F_{2}$. This is what we wanted to demonstrate.

\section{QUICK SUMMARY}

1. This paper is intended to supplement the companion paper entitled Identity Analytics and Belief Structures (Katzan 2010) in the area of compatibility relations, which is the mapping between two frames of discernment.

2. Introductory material on identity and belief is covered.

3. A technical description of uncertainty theory is given.

4. Compatibility relations as they apply to identity analysis are described.

5. Subordinate topics are covered including evidence, nodes, links, and decision scripts are covered.

6. Numerous examples are given.

\section{AUTHOR INFORMATION}

Dr. Harry Katzan is the author of books and papers on computer science, decision science, and service science and is the founding editor of the Journal of Service Science. His current research interests are in the area of a strategy for trusted identity in cyberspace.

\section{REFERENCES}

1. Campbell, J. 1989. The Improbable Machine, New York: Simon \& Schuster, Inc.

2. Dempster, A.P. 1967, Upper and Lower Probabilities Induced by a Multivalued Mapping. The Annals of Statistics 28:325-339.

3. Katzan, H. 1992. Managing Uncertainty: A Pragmatic Approach, New York: Van Nostrand Reinhold Co.

4. Katzan, H. 2008. Categorical Analytics Based on Consensus Theory. Journal of Business and Economics Research, 6(8), 89-102. 
5. Katzan, H. 2010. Identity Analytics and Belief Structures. Journal of Business and Economics Research, 8(6), 31-39.

6. Neapolitan, R. 1990. Probabilistic Reasoning in Expert Systems: Theory and Applications, New York: John Wiley \& Sons, Inc.

7. Russell, B. 1912. The Problems of Philosophy. (Republished by Barnes \& Noble, New York, 2004).

8. Russell, B. 1919. Introduction to Mathematical Philosophy. (Republished by Barnes \& Noble, New York, 2005).

9. Shafer, G. 1976, A Mathematical Theory of Evidence, Princeton, NJ: Princeton University Press.

10. Stroll, A. 1967. Identity. (Entry in The Encyclopedia of Philosophy, Volume 4, Paul Edwards, Editor-inChief, New York: Macmillan Publishing Co., 1967).

11. Sutherland, J. 1975. Systems: Analysis, Administration, and Architecture, New York: Van Nostrand Reinhold Co.

12. Tversky, A. and D. Kahneman. 1974. Judgment under Uncertainty: Heuristics and Biases. Science 185 (September 27, 1974), 1124-1131.

13. Zadeh, L. 1986. A Simple View of the Dempster-Shafer Theory of Evidence and Its Implication for the Rule of Combination. The AI Magazine (Summer 1986), pp. 85-90. 